\title{
EMDR IN OBSESSIVE-COMPULSIVE DISORDER: A CASE REPORT
}

\author{
Seghaier S., Ben Cheikh C., Lebdi M., Jouini R., El Kefi H., Oumaya A. \\ Military Hospital of Tunis, Tunisia
}

\section{Background:}

Although there have been several case studies reporting the successful eye movement desensitization and reprocessing (EMDR) treatment of specific phobias, EMDR has been considered a viable treatment for posttraumatic stress disorder and little research has been done on this application.

\section{Objectives:}

In the current study, we aimed to evaluate the impact of EMDR as a treatment for obsessive-compulsive disorder (OCD) in a patient diagnosed with OCD and who was symptomatic despite medical treatment and cognitive behavioural therapy.

\section{Material and methods:}

A female patient with treatment resistant OCD, who had shown no response to pharmacological or psychotherapeutic interventions, was followed in our psychiatry department in The Military Hospital of Tunis. An adaptation of Shapiro's (2001) phobia protocol was developed, based on the theoretical view that OCD is a selfperpetuating disorder, with OCD compulsions and obsessions and current triggers reinforcing and maintaining the disorder. The protocol adaptation begins by addressing current obsessions and compulsions, instead of working on past memories. The strategy delays the cognitive installation phase in the desensitization of triggers.

\section{Results:}

The lady was 58 year-old and met the DSM V criteria for OCD. She showed excessive nervousness due to contamination obsessions and excessive cleaning and hand washing compulsions as well as anxiety and avoidances. During her treatment, she received a total of 12 EMDR sessions. Each session lasted between 60-90 minutes, and was assessed with the Yale-Brown Obsessive Compulsive Scale (Y-BOCS), with a pretreatment score in the extreme range. Within the fifth session, the lady disclosed a past repetitive trauma consisting of spitting on her face every time she made a behavior deemed inappropriate by her mother. The Y-BOCS scores showed substantial decreases and the lady reported significant symptom improvement, with the treatment effects maintained at 4-6 months follow-up.

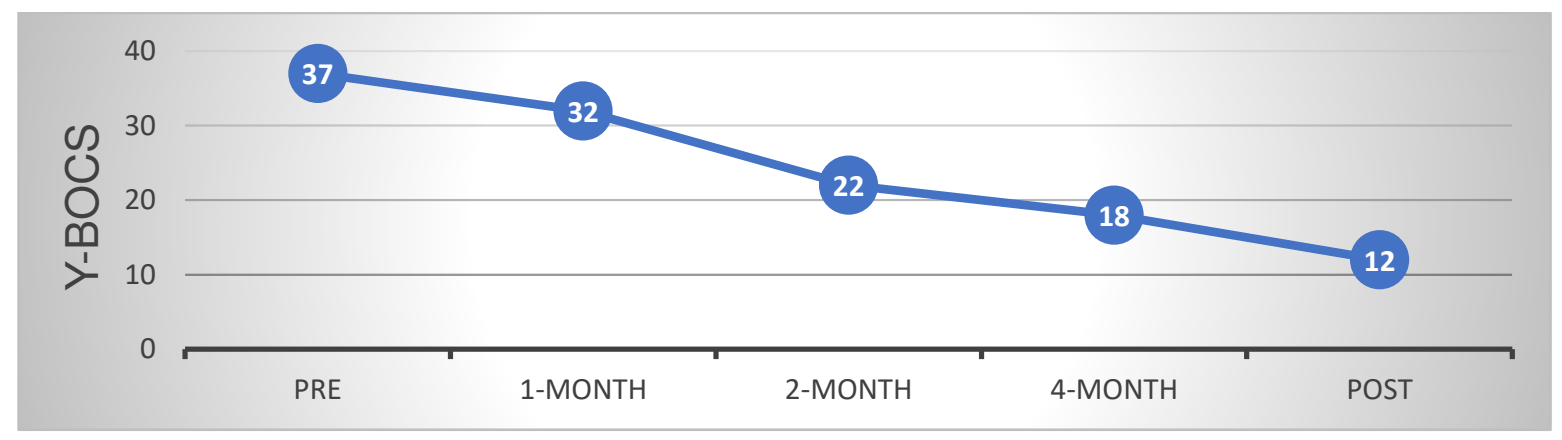

Figure 1: Y-BOCS scores during follow-up

\section{Conclusions:}

Our study demonstrated that the adapted EMDR phobia protocol is applicable in treatment resistant OCD and that trauma in the patient's past may be directly linked to the onset of this psychological disorder. This is based on the main theoretical principle of the EMDR model that posits that resolving disturbing memories will resolve related symptoms (Shapiro, 2001). However, further researches are recommended to better understand how EMDR phobia protocol can be applied in the treatment of specific fears and phobic conditions.

Keywords: Trauma; Eye Movement Desensitization and Reprocessing; Obsessive-Compulsive Disorder; Post-Traumatic Stress Disorder. 